\title{
Configuration Selection for Reconfigurable Control of Piecewise Affine Systems
}

\author{
Tabatabaeipour, Mojtaba; Gholami, M.; Bak, T.
}

Published in:

International Journal of Control

Link to article, DOI:

$10.1080 / 00207179.2015 .1006684$

Publication date:

2015

Document Version

Peer reviewed version

Link back to DTU Orbit

Citation (APA):

Tabatabaeipour, M., Gholami, M., \& Bak, T. (2015). Configuration Selection for Reconfigurable Control of Piecewise Affine Systems. International Journal of Control, 88(6), 1310-1323.

https://doi.org/10.1080/00207179.2015.1006684

\section{General rights}

Copyright and moral rights for the publications made accessible in the public portal are retained by the authors and/or other copyright owners and it is a condition of accessing publications that users recognise and abide by the legal requirements associated with these rights.

- Users may download and print one copy of any publication from the public portal for the purpose of private study or research.

- You may not further distribute the material or use it for any profit-making activity or commercial gain

- You may freely distribute the URL identifying the publication in the public portal

If you believe that this document breaches copyright please contact us providing details, and we will remove access to the work immediately and investigate your claim. 


\title{
Configuration Selection for Reconfigurable Control of Piecewise Affine Systems
}

\author{
S. M. Tabatabaeipour ${ }^{a},{ }^{*}$ M. Gholami ${ }^{b}$, T. Bak $^{b}$ \\ ${ }^{a}$ Section for Automation and Control, Department of Electrical Engineering, \\ Technical University of Denmark, Kgs. Lyngby, DK-2800, Denmark \\ ${ }^{b}$ Department of Electronic Systems, Aalborg University, DK-9220, Denmark
}

\begin{abstract}
In this paper, the problem of configuration selection i.e. sensor/actuator placement for piecewise affine (PWA) systems subject to both sensor and actuator faults is considered. A method is proposed that provides a tool for the design phase to decide about the optimal placement of sensor/actuators where the reconfigurability of the system subject to sensor and actuator faults is also taken into account. Using a lattice of possible configurations (sensor/actuator placements), the reconfigurability of the system subject to faults for each configuration is evaluated and based on that one can draw conclusions about the reconfigurability of the system and the optimal configuration in the architecture design phase. A reconfigurable control must ensure stability of the reconfigured system and, if possible, a graceful degradation in the performance. Therefore, in the proposed reconfigurability analysis, we consider both stabilizability and performance of the system. The efficiency of the proposed method is demonstrated on several numerical examples.
\end{abstract}

\section{INTRODUCTION}

Performance of a modern control system typically relies on a number of strongly interconnected components. A fault in a component may degrade the performance of the system or even result in the loss of its functionality or stability. In many cases it may even result in hazardous events. Due to increasing demands on the safety and reliability in modern industrial systems, it is desirable to develop control systems that can tolerate component malfunctions while preserving the stability and functionality of the system and providing a desirable performance. Such controllers are called fault tolerant.

Fault tolerant control (FTC) systems are generally divided into two categories:passive (PFTC) and active (AFTC). In PFTC, the structure of the system is fixed and pre-designed such that it can tolerate a set of

\footnotetext{
${ }^{* *}$ Corresponding author. Email: setaba@.elektro.dtu.dk
} 
faults. In this case the fault tolerant controller is a common solution to a set of control problem including the faulty and the normal plant. In AFTC, a specific controller is designed for each faulty case. The fault is detected using a fault detection and diagnosis (FDD) scheme. Then, based on the information from the FDD module, the controller is re-designed such that the overall stability of the system is preserved and an acceptable performance is provided (see Figure 1). If the set of sensors and actuators used for control does not change and only the controller parameters are modified the control re-design is called faultaccommodation. However, in the case of severe faults, when the set of measurements and actuators used for control as well as the controller parameters and/or structure are changed, then the re-design step is called control reconfiguration.

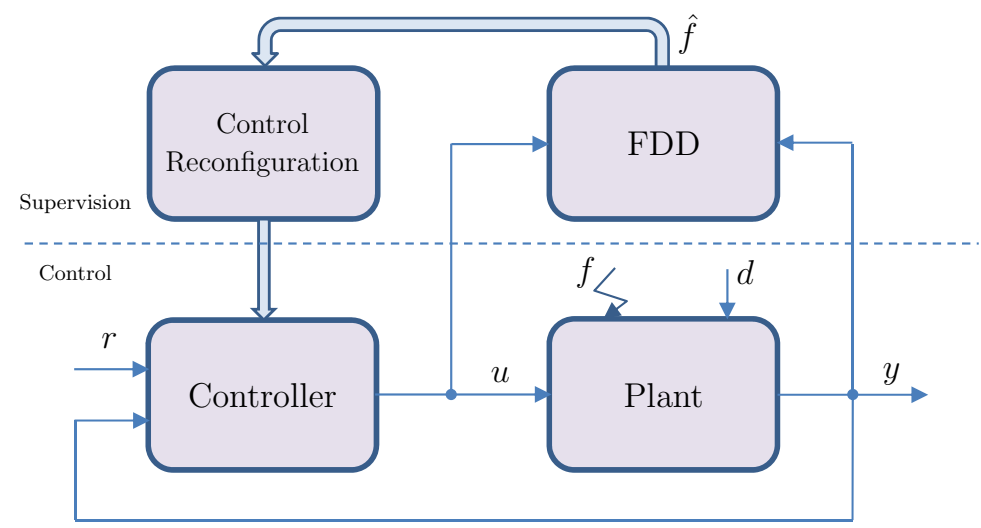

Figure 1: Structure of Active Fault Tolerant Control

In a control reconfiguration problem, the reconfigured controller must be able to recover specific properties of the nominal closed-loop system such as stability, performance, etc. despite a fault such as loss of an actuator or a sensor. The exact recovery of the performance is not usually achievable; hence a graceful degradation is desirable. In the configuration selection problem, sensor and actuators must be placed such that some given properties and requirement for the closed-loop system are satisfied. When reconfigurability subject to a set of faults is also considered in the configuration selection, sensor and actuators must be placed such that the system is reconfigurable despite occurrence of the given set of faults and the reconfigured system is stable and provides a specified performance. For example, if an actuator is so crucial that its loss would result in an unsatisfactory performance then it is recommended in the architecture design phase, to add a redundant actuator so that the system can tolerate loss of this actuator. However, the final decision depends on many factors including the cost of adding another actuator and the loss due to shut down or instability because of the fault in that actuator. Obviously, if the system is safety-critical, the latter cost is substantial. In this paper, we address the problem of configuration selection for piecewise affine systems where the reconfigurability subject to sensor and actuator faults is also taken into account.

To address the problem, we extend the concept of reconfigurability subject to a fault to PWA systems. 
Reconfigurability is the capability of the system to preserve some properties, e.g. stability or performance, of the system when a fault has occurred. If the system is reconfigurable subject to a fault, it means that, we can design a specific controller for the specified fault that can preserve stability and provide an admissible performance. In the operation when this fault happens the AFTC will reconfigure the controller when the fault is detected. If the system is not reconfigurable subject to the specified fault, then the specified fault cannot be tolerated and either the system would be unstable or its performance would degrade to an unacceptable level. In this case, some other appropriate actions such as system shut-down should be performed.

Reconfigurability analysis in the configuration selection phase, provides us a helpful insight about the optimal sensor and actuator placement as well as dependency of the system performance on each sensor or actuator. This helps us to decide where we should use hardware redundancy and analytical redundancy to design an efficient reconfigurable controller with a better performance and a lower cost.

Reconfigurability of linear time invariant systems is measured by controllability and observability Grammians in Frei et al. (1999). A measure for control reconfigurability of linear systems is proposed in Wu et al. (2000). The smallest second-order mode is used as a measure for reconfigurability of the system to preserve an acceptable performance in the presence of a fault. In Staroswiecki (2002), the fault tolerant property of a configuration with respect to an actuator fault is investigated. Two cases are considered. In the first case, only achieving the control objective is considered, but in the second case the control objective must be achieved and the control energy must be admissible. The method uses a Grammian based approach. This result is extended to the admissibility of a linear quadratic cost function in Staroswiecki (2003). Khelassi et al. (2009) defines reconfigurability of the system not only based on the controllability Grammian, but also based on the system reliability. While in the aforementioned methods, the reconfigurability measures are computed off-line, an online method for calculation of the controllability Grammian using input/output data is proposed in Gonzalez-Contreras et al. (2009).

All of the aforementioned methods are for linear systems. Many of the complex industrial systems either exhibit nonlinear behavior or contain both discrete and continuous components. An attractive modeling framework for such systems is the framework of piecewise affine systems (PWA). This is because PWA framework proposes an efficient way to describe the dynamic of systems exhibiting switching between a number of linear systems where switching is state-dependent Johansson (2003); Heemels et al. (2001). In many nonlinear systems, this switching is because of PWA components such as dead-zone, saturation, hysteresis etc. These nonlinearities appear in many industrial applications and can be efficiently modeled by a PWA system. For example, in van de Wouw and Pavlov (2008) it is shown that many practical systems such as mechanical motion systems with friction can be efficiently modeled as PWA systems. Moreover, PWA systems can approximate nonlinear systems effectively Richter et al. (2011). Also, system identification methods such as Tabatabaeipour et al. (2006), Tabatabaeipour et al. (2006), Ferrari-Trecate et al. (2003), and Ren et al. (2012) can be used to identify a PWA model of a nonlinear system. For PFTC and AFTC of PWA system see Tabatabaeipour et al. (2012), Richter et al. (2011) and Tabatabaeipour and Bak (2014) and references therein. 
In Yang (2006) reconfigurability of a class of linear switched systems is considered. Reconfigurability is defined as the controllability of the system and an algebraic approach for reconfigurability is given. In Tabatabaeipour et al. (2011), we considered reconfigurability of PWA systems against actuator faults, where only complete loss of actuator gain is considered. A system subject to a fault is called reconfigurable if it is not only stabilizable using a state feedback control law, but also the performance cost of the systems is admissible with any initial condition in a given bounded region. In other words, we have considered both stability and admissibility of the performance of the system as a criteria for reconfigurability.

In this work we consider the problem of configuration selection for designing a reconfigurable control architecture for PWA systems. We extend the notion of reconfigurability introduced in Tabatabaeipour et al. (2011). We consider both actuator and sensor faults. Instead of using state feedback, static output feedback is used. For the performance, both quadratic cost and $H_{\infty}$ performance are considered. A configuration subject to a sensor and/or actuator fault is called reconfigurable if there exist a static output feedback that stabilizes the system and the performance of the system (quadratic cost or the $H_{\infty}$ performance) is admissible. The problem is cast as the feasibility of a convex optimization problem with LMI constraints. The optimization problem can be solved efficiently using available softwares such as YALMIP/SeDuMi or LMILAB. Using the proposed reconfigurability analysis with the lattice of configurations, we can evaluate criticality of each sensor and actuators and decide about its required hardware redundancy, reliability, maintenance policy etc. . The proposed method provides a tool that can be used in the design phase to decide about the optimal placement of sensor/actuators where the reconfigurability of the system subject to sensor and actuator faults is also taken into account.

The paper is organized as follows. In Section II, the PWA model and actuator and sensor faults are given. In Section III, reconfigurability with respect to quadratic performance cost is defined and sufficient conditions for reconfigurability are given. In Section IV reconfigurability with respect to $H_{\infty}$ performance is defined and sufficient conditions for it are derived. Section IV is dedicated to the simulation results for the climate control system and two numerical example. The conclusion is presented in the Section V.

\section{Piecewise Affine systems and actuator and sensor fault models}

\subsection{Piecewise Affine Systems}

We consider a PWA discrete time system of the following form:

$$
\begin{gathered}
x(k+1)=A_{i} x(k)+B_{i} u(k)+b_{i} \\
y(k)=C_{i} x(k) \quad \text { for } x(k) \in \mathcal{R}_{i}, i \in \mathcal{I},
\end{gathered}
$$

where $x(k) \in \mathbb{R}^{n}$ is the state, $u(k) \in \mathbb{R}^{m}$ is the control input, and $y(k) \in \mathbb{R}^{p}$ is the measured output. $\left\{\mathcal{R}_{i}\right\}_{i=1}^{s} \subseteq \mathbb{R}^{p}$ denotes a partition of the state space into a number of polyhedral regions $\mathcal{R}_{i}, i \in \mathcal{I}=$ $\{1, \cdots, s\}$. Each polyhedral region is given by:

$$
\mathcal{R}_{i}=\left\{x \mid H_{i} x \leq h_{i}\right\} .
$$


The set $\mathcal{I}$ is partitioned to $\mathcal{I}_{0} \cup \mathcal{I}_{1}$, where $\mathcal{I}_{0}$ denotes the index set of subsystems that contain the origin and $\mathcal{I}_{1}$ is the index set of the subsystems that do not contain the origin. It is assumed that $b_{i}=0$ for $i \in \mathcal{I}_{0}$.

Each polyhedral region $\mathcal{R}_{i}$ can be over-approximated with a union of $\ell_{i}$ ellipsoids, i.e:

$$
\mathcal{R}_{i} \subseteq \bigcup_{j=1}^{\ell_{i}} \mathcal{E}_{i j},
$$

where each ellipsoid is represented by the matrix $E_{i j}$ and the scalar $f_{i j}$ such that $\mathcal{E}_{i j}=\left\{x \mid\left\|E_{i j} x+f_{i j}\right\| \leq 1\right\}$, see Rodrigues and Boyd (2005). This approximation is used in this paper to deal with the affine term for subsystems with $i \in \mathcal{I}_{1}$ which helps us to cast the control problem in terms of LMIs.

All possible switchings from region $\mathcal{R}_{i}$ to $\mathcal{R}_{j}$ are represented by the set $\mathcal{S}$ :

$$
\mathcal{S}:=\left\{(i, j) \mid x(k) \in \mathcal{R}_{i}, x(k+1) \in \mathcal{R}_{j}\right\} .
$$

The set $\mathcal{S}$ can be computed using reachability analysis for piecewise affine systems. A conservative approach is to assume that switching happens between all subspaces i.e $\mathcal{S}=\mathcal{I} \times \mathcal{I}=\{(i, j) \mid i, j \in \mathcal{I}\}$.

\section{Configurations and Faults}

We consider a system with a set of actuators and sensors given by $F_{0}$. The cardinality of this set is denoted by $\operatorname{card}\left(F_{0}\right)$. A configuration $F_{i}$ is a subset of $F_{0}$ meaning that a subset of actuators and sensors are selected as input and output of the system. The set of all possible configurations generated by $F_{0}$ is the power set of $F_{0}$ denoted by $\mathcal{P}\left(F_{0}\right)$. If we equip the power set $\mathcal{P}\left(F_{0}\right)$ with the set-inclusion partial ordering then we have a lattice that is denoted by $L\left(F_{0}\right)$. The lattice is usually represented by a non-directed graph where configuration are its vertices and and there is an edge between two vertices (two configurations) $F_{i}$ and $F_{j}$ if they differ only in one component i.e. there exist an actuator or a sensor that belongs to $F_{i}$ but not $F_{j}$ or vice versa:

$$
\exists \sigma \in F_{0}: F_{i}=F_{j} \cup\{\sigma\} \text { or } F_{j}=F_{i} \cup\{\sigma\} .
$$

We can organize the graph into levels, $l_{i}$, such that each level contains those configurations with the same number of components. The full configuration is generally the top level card $\left(F_{0}\right)$ and the empty configuration is the bottom level, see (Staroswiecki et al., 2012).

\subsubsection{Example}

Consider a system with 3 actuators $\left\{a_{1}, a_{2}, a_{3}\right\}$ and two sensors $s_{1}, s_{2}$. The lattice of system configurations is shown in Figure 2.

\subsection{Fault Model}

In this work, we consider both actuator and sensor faults. A fault in an actuator is an event that changes the input matrix $B_{i}$ of the system to $B_{i}^{f}$. A total loss of an actuator is represented by removing the corresponding 


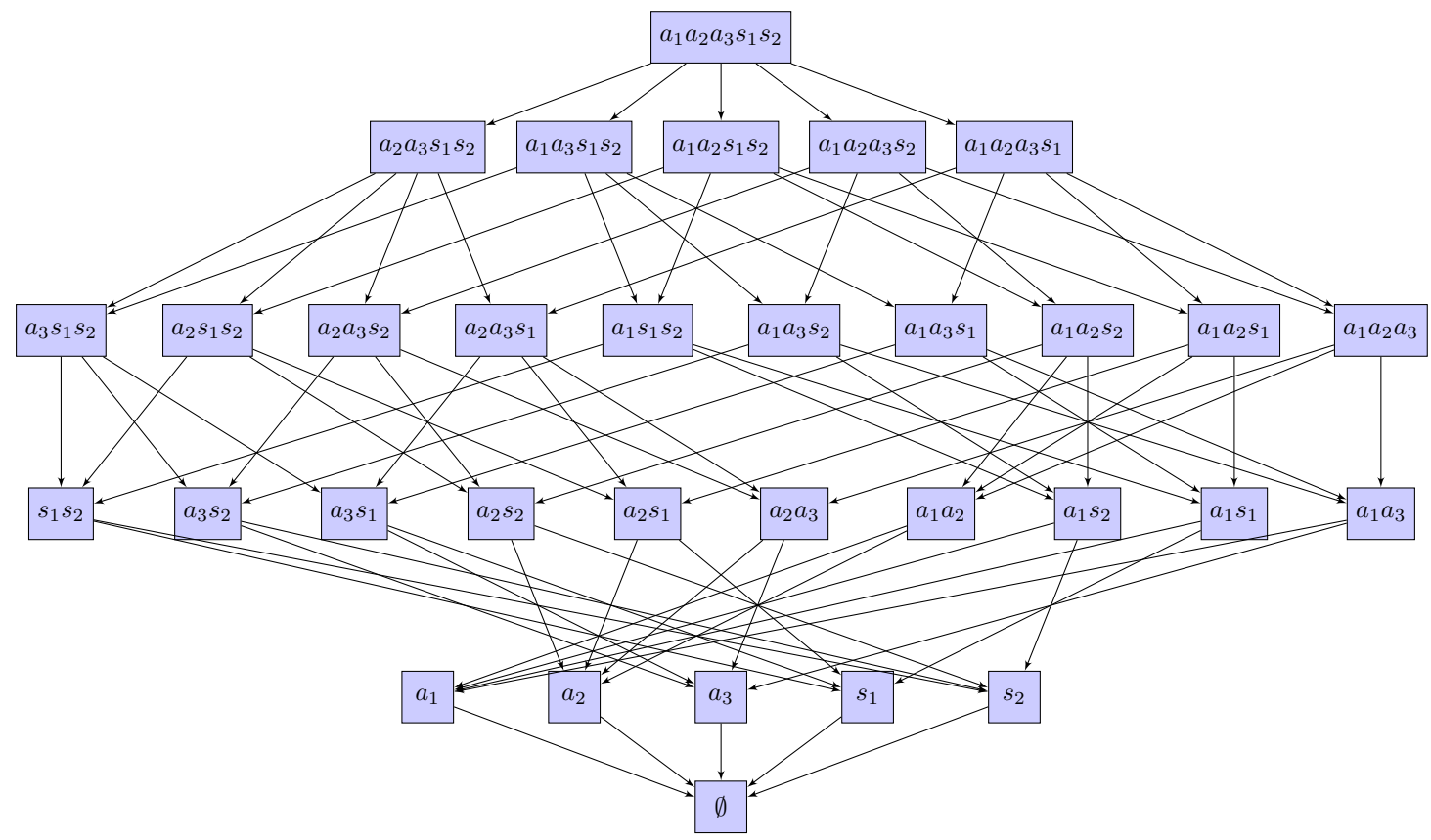

Figure 2: Lattice of possible configurations for a system with three actuators $a_{1}, a_{2}, a_{3}$ and two sensors $s_{1}, s_{2}$. Moving from top of the diagram to its bottom represents the loss of components.

column of $B_{i}$. Similarly, a fault in a sensor is an event that changes the output matrix of the system $C_{i}$ to $C_{i}^{f}$. A total loss of a sensor is represented by removing the corresponding row of $C_{i}$.

In view of the system configurations graph, a loss of a component (sensor or actuator) changes the configuration of the system from configuration $F$ to $F^{\prime}$ such that $F^{\prime}$ belongs to one level lower than that of $F$ i.e. if $F$ belongs to the level $l_{F}$, then $l_{F \prime}=l_{F}-1$. Therefore, moving from top of the diagram of the lattice of configurations to its bottom represents the loss of components (See Figure 2).

Without loss of generality, it is assumed that $C_{i}^{f}, i=1,2, \ldots, s$ is of full column (or row) rank. Then, there exist nonsingular transformation matrices $T_{c f i}, i=1,2, \ldots, s$, such that

$$
C_{i}^{f} T_{c f i}=\left[\begin{array}{ll}
I & 0
\end{array}\right] .
$$

A special solution for $T_{c i}$ can be obtained by

$$
T_{c f i}=\left[\begin{array}{ll}
C_{i}^{f^{T}} C_{i}^{f} C_{i}^{f^{T}} & C_{i}^{f^{\perp}}
\end{array}\right] .
$$

where $(\bullet)^{\perp}$ denotes an orthogonal basis for the null space of $(\bullet)$. 


\section{Static Output Feedback Design for PWA systems}

\subsection{Piecewise Quadratic Stability}

The problem of static output feedback design is to design a static output feedback of the form:

$$
u(k)=K y(k)
$$

such that the closed loop PWA system

$$
x(k+1)=\mathbf{A}_{i} x(k)+b_{i},
$$

where $\mathbf{A}_{i}=A_{i}+B_{i} K C_{i}$, is exponentially stable.

\subsection{PWL Quadratic Regulator (PWLQR)}

The aim of the control design problem is to design a controller of the form (9) such that it stabilizes the system and provides an upper bound on the following quadratic cost function associated with the system:

$$
J=\sum_{k=0}^{\infty} x^{T}(k) Q_{i} x(k)+u^{T}(k) R_{i} u(k),
$$

where $Q_{i} \geq 0$ and $R_{i} \geq 0$ are given weighting matrices of appropriate dimensions. The PWA system subject to a fault is called reconfigurable if a static output feedback controller can be found that stabilizes the system and the upper bound on the quadratic cost is less than a pre-specified threshold.

Definition 1. The system (2) subject to fault $f$ is called reconfigurable if there exist a static output feedback control law of the form (9) which stabilizes the system and the upper bound on the cost function (11) is admissible i.e. is less than a specified given threshold.

The following, gives sufficient conditions for a PWA systems to be stabilizable by a static output feedback controller.

Theorem 1. If there exist symmetric matrices $X_{i}=X_{i}^{T}>0$, matrices $U$, positive constants $\mu_{i l}>0$, and matrices $G_{i}$ with the following structure

$$
G_{i}=\left[\begin{array}{cc}
G_{11} & 0 \\
G_{i 21} & G_{i 22}
\end{array}\right]
$$


such that:

$$
\begin{gathered}
{\left[\begin{array}{ccc}
X_{i}-\bar{G}_{i}-\bar{G}_{i}^{T} & * & * \\
\left(A_{i} \bar{G}_{i}+B_{i}^{f}\left[\begin{array}{ll}
U & 0
\end{array}\right]\right) & -X_{j}-\mu_{i l} b_{i} b_{i}^{T} & * \\
E_{i l} \bar{G}_{i} & -\mu_{i l} f_{i l} b_{i}^{T} & -\mu_{i l}\left(f_{i l} f_{i l}^{T}-1\right)
\end{array}\right]<0,} \\
\forall(i, j) \in \mathcal{S}, i \in \mathcal{I}_{1}, l=1, \ldots, \ell_{i}, \\
{\left[\begin{array}{cc}
X_{i}-\bar{G}_{i}-\bar{G}_{i}^{T} & * \\
\left(A_{i} \bar{G}_{i}+B_{i}^{f}\left[\begin{array}{ll}
U & 0
\end{array}\right]\right)^{T} & -X_{j}
\end{array}\right]<0,} \\
\forall(i, j) \in \mathcal{S}, i \in \mathcal{I}_{0},
\end{gathered}
$$

with $\bar{G}_{i}=T_{\text {cif }} G_{i}$, then there exist a static output feedback control law of the form (9) for the PWA system such that the closed loop system is exponentially stable. The piecewise linear feedback gains are given by:

$$
K=U G_{11}^{-1} .
$$

Proof. See A.1.

The above theorem only considers stability. In many situations, the system might be stabilizable but the cost of reaching to the origin from the initial state might not be admissible. To include admissibility of the upper bound on the cost function we introduce the following theorem.

Theorem 2. If there exist symmetric matrices $X_{i}=X_{i}^{T}>0$ and matrices $U_{i}$, positive constants $\mu_{i l}>0$, and matrices $G_{i}$ with the following structure

$$
G_{i}=\left[\begin{array}{cc}
G_{11} & 0 \\
G_{i 21} & G_{i 22}
\end{array}\right]
$$

such that: 


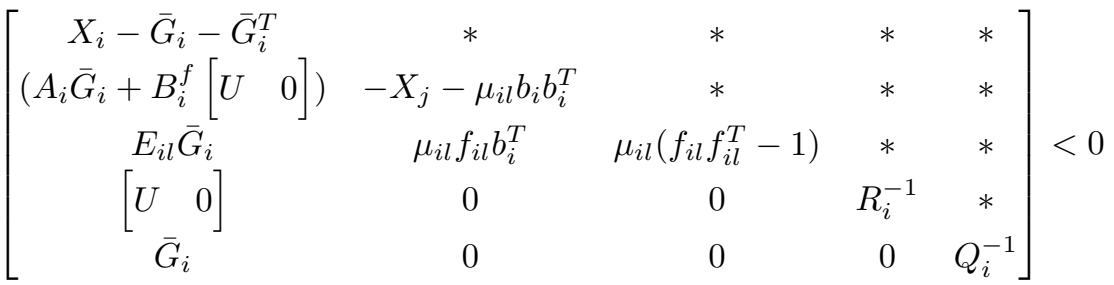

$$
\begin{aligned}
& \forall(i, j) \in \mathcal{S}, i \in \mathcal{I}_{1}, l=1, \ldots, \ell_{i},
\end{aligned}
$$

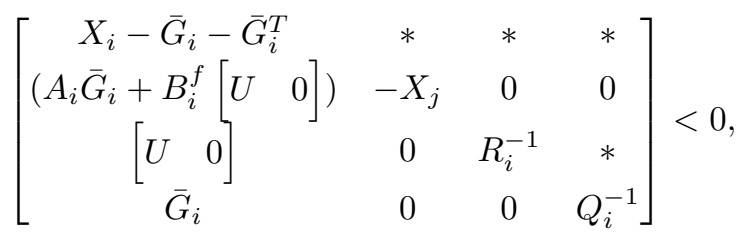

$$
\begin{aligned}
& \forall(i, j) \in \mathcal{S}, i \in \mathcal{I}_{0},
\end{aligned}
$$

with $\bar{G}_{i}=T_{c f i} G_{i}$, then there exist a SOF control law of the form (9) for the PWA system (2) subject to the fault $f$ such that the closed loop system is exponentially stable. The PWL feedback gains are given by:

$$
K=U G_{11}^{-1},
$$

and the upper bound on the cost function (11) satisfies:

$$
J \leq x(0)^{T} X_{i_{0}}^{-1} x(0)
$$

where $i_{0}$ is the region index for the initial condition, i.e. $y(0) \in \mathcal{R}_{i_{0}}$.

Proof. See A.2.

The upper bound found in the theorem (2) is not optimal. We are interested to minimize this cost to find a controller with the minimum cost. The upper bound of (11), could be minimized in the following way. The initial condition is considered as a random variable with uniform distribution in a bounded region $\overline{\mathcal{X}}$. Then, it is tried to minimize the expected value of the cost function. We have:

$$
E(J) \leq E\left(\operatorname{tr}\left(P_{i_{0}} x(0) x^{T}(0)\right)\right) \leq \sum_{i \in \mathcal{I}} \sigma_{i} \operatorname{tr}\left(P_{i} L_{i}\right),
$$

where $L_{i}=E\left(x(0) x^{T}(0)\right)$ is the expectation of $x(0) x^{T}(0)$ corresponding to $x(0) \in \mathcal{X}_{i}, i \in \mathcal{I}, \operatorname{tr}(\cdot)$ is the trace 
operator and $\sigma_{i}$ is the probability of $x(0) \in \mathcal{X}_{i}$. Then, the optimization problem is:

$$
\begin{gathered}
J^{*}=\min _{X_{i}, U_{i}, V_{i}} \sum_{i \in \mathcal{I}} \sigma_{i} \operatorname{tr}\left(X_{i}^{-1} L_{i}\right) \\
\text { s.t. }\left\{\begin{array}{c}
(18) \\
(19) \\
X_{i}=X_{i}^{T}>0,
\end{array}\right.
\end{gathered}
$$

The above optimization problem is non-convex. To convert it to a convex optimization problem, we introduce new variables $Y_{i}, i \in \mathcal{I}$, which satisfies:

$$
\left[\begin{array}{cc}
Y_{i} & I \\
I & Z_{i}
\end{array}\right] \geq 0 .
$$

Using Schur complement, the above constraint is equivalent to $Z_{i}^{-1} \leq Y_{i}$. Therefore, the objective function in (24), which is nonlinear in term of $Z_{i}$, can be converted to $\sum_{i \in \mathcal{I}} \sigma_{i} \operatorname{tr}\left(Y_{i} L_{i}\right)$. Consequently, the optimization problem (24) can be transformed to the following convex form:

$$
\begin{array}{r}
J^{*}=\min _{X_{i}, U_{i}, V_{i}, Y_{i} \epsilon_{i}} \sum_{i \in \mathcal{I}} \sigma_{i} \operatorname{tr}\left(Y_{i} L_{i}\right) \\
\text { s.t. }\left\{\begin{array}{c}
(18), \\
(19), \\
(25), \\
X_{i}=X_{i}^{T}>0,
\end{array}\right.
\end{array}
$$

In the following theorem we consider the properties for reconfigurability to be stability and admissibility of the optimal upper bound on the cost function.

Theorem 3. The system (2) subject to fault $f$ with respect to admissibility threshold $\bar{J}$ on the cost function (11) is reconfigurable if:

- (18) and (19) are satisfied,

- $J^{*}<\bar{J}$.

Proof. Satisfaction of (13) and (14) guarantees that the system is stabilizable with a SOF controller and satisfying $J^{*}<\bar{J}$ is equal to admissibility of the cost. Therefore, based on definition 1 the system subject to fault $f$ is reconfigurable. 


\subsection{Example 1}

In this section we consider the following PWA system:

$$
\begin{gathered}
A_{1}=\left[\begin{array}{lll}
0.1509 & 0.8600 & 0.4966 \\
0.6979 & 0.8537 & 0.8998 \\
0.3784 & 0.5936 & 0.8216
\end{array}\right] \\
A_{2}=\left[\begin{array}{lll}
0.6449 & 0.3420 & 0.5341 \\
0.8180 & 0.2897 & 0.7271 \\
0.6602 & 0.3412 & 0.3093
\end{array}\right], \\
A_{3}=\left[\begin{array}{lll}
0.8385 & 0.7027 & 0.6946 \\
0.5681 & 0.5466 & 0.6213 \\
0.3704 & 0.4449 & 0.7948
\end{array}\right], \\
B_{1}=\left[\begin{array}{l}
1 \\
1 \\
1
\end{array}\right], B_{3}=B_{2}=B_{1}, \\
b_{1}=\left[\begin{array}{l}
0.5 \\
0.5 \\
0.5
\end{array}\right], b_{3}=b_{1}, b_{2}=\left[\begin{array}{l}
0 \\
0 \\
0
\end{array}\right] .
\end{gathered}
$$

The system is assumed to be a slab system where switching is based on the first state. i.e:

$$
\begin{gathered}
\mathcal{R}_{1}=\left\{x_{1} \mid-6 \leq x_{1} \leq-3\right\}, \\
\mathcal{R}_{2}=\left\{x_{1} \mid-3 \leq x_{1} \leq-3\right\}, \\
\mathcal{R}_{3}=\left\{x_{1} \mid 3 \leq x_{1} \leq 6\right\} .
\end{gathered}
$$

For the quadratic cost performance parameters are chosen as:

$$
Q=0.2 I_{3 \times 3}, \quad R=0.2
$$

We use reconfigurability analysis to see the effect of actuator and sensor faults on the reconfigurability of the system and to decide which measurements are more important for designing a fault tolerant control system. Figure 3 shows the result of reconfigurability analysis on the lattice of systems configurations when only stabilizability is considered. Since we only have one actuator, in lattice only sensor faults are considered. At the first level, it is assumed that we can measure all states. At the second level one of the sensors is faulty and at the third level, two of the sensors are subject to outage faults. A white node means the the system is stabilizable and a grey node shows that the system is not stabilizable with the corresponding configuration. Therefore, the analysis of the lattice suggest that to be able reconfigure the system subject to one sensor fault, we need at least two sensors which must include a measurement of the first state. In other words, if we have redundancy in sensor 1 , the system can be reconfigured when a sensor is lost. In case $s_{2}$ or $s_{3}$ fails, the 
system can be reconfigured using analytical redundancy, and if $s_{1}$ fails, we have to use hardware redundancy. Table 1 shows the result of the reconfigruability analysis the performance cost of the system is also considered.

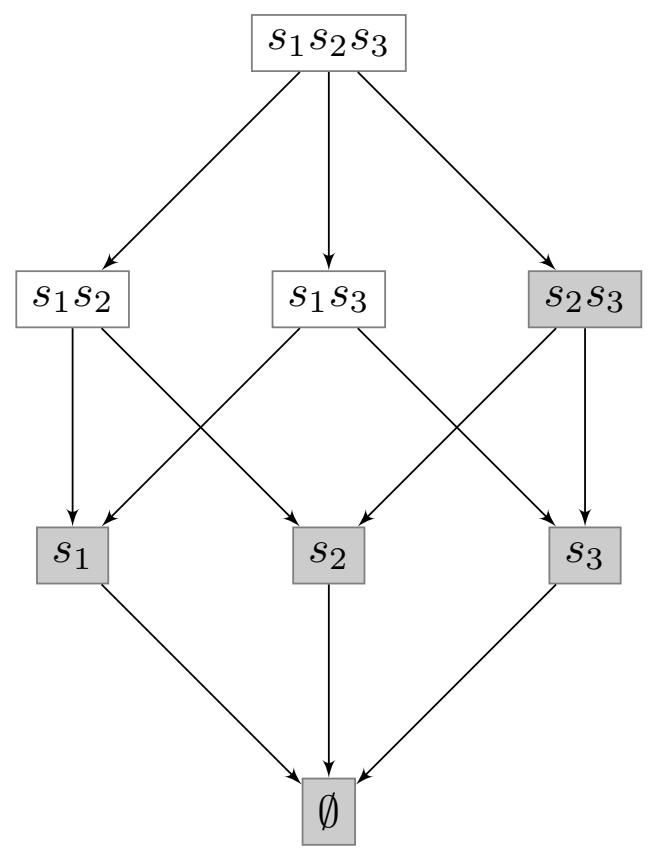

Figure 3: Lattice of system configurations for example 1

Only stabilizable configurations are shown in the table. The first row shows when the weighting matrix $Q_{i}$ 's are chosen as $\operatorname{diag}\{0.2,0.2,0.2\}$ and the second row shows when $Q_{i}$ 's are chosen as $\operatorname{diag}\{0.2,0.1,0.1\}$. As can be seen, even though all of these configurations are stabilizable, but the performance of the system varies a lot. For example, if the admissible performance cost is 19 , then the system with a fault in sensor 3 is not reconfigurable when $Q_{i}$ 's are chosen as $\operatorname{diag}\{0.2,0.1,0.1\}$. This means that sensors 1 and 3 are of crucial importance and it is important to ensure hardware redundancy for them, because if we lose sensor 1 , then the system is not stabilizable, and if we lose sensor 3, even thought the system is stabilizable, the performance of the system is not admissible.

Table 1: Stabilizable configurations and associated quadratic cost

\begin{tabular}{|c|c|c|c|}
\hline Weighting matrices & \multicolumn{3}{|c|}{ sensor configurations } \\
\cline { 2 - 4 }$Q_{i}$ & $\{1,2,3\}$ & $\{1,3\}$ & $\{1,2\}$ \\
\hline $\operatorname{diag}\{0.2,0.2,0.2\}$ & 6.56 & 9.09 & 18.53 \\
$\operatorname{diag}\{0.2,0.1,0.1\}$ & 7.6 & 9.59 & 19.1 \\
\hline
\end{tabular}




\subsection{Probabilistic Measures}

In this section we discuss how we can use the lattice of configurations with the failure rates of components to drive probabilistic measures for reliability of the system. We assume that the failure rates of each sensor and actuators are given and they have a exponential distribution with the occurrence rate $\lambda_{a_{i}}$ or $\lambda_{s_{i}}$. It is also assumed that the failure event for each components are independent. Here, we do not consider the repair rates, but the framework allows to consider probabilistic repairs with exponential distributions with given rates. We also assume that the rate of failure of the fault-tolerant control systems (including the FDI and control reconfiguration module) is sufficiently smaller that rates of failure of sensors or actuators and therefore it can be neglected in our analysis. Using the failure rates information with the lattice of all configurations, a continuous time Markov chain is constructed such that the transition rate between two configurations $F_{i}$ and $F_{j}$ is nonzero if there is an edge connecting these two vertices. Then, the transition rate between between $F_{i}$ and $F_{j}$ is determined by the rate of failure of the only component that they differ which is denoted by $\lambda_{i j}$ here. Note that in this way the non-directional graph of the lattice of configurations becomes a one-directional graph and if we consider repairs it becomes a bi-directional graph. Therefore, one can construct a continuous-time Markov chain with discrete state $X$ taking values in $\mathcal{F}=\left\{1, \cdots, 2^{N_{a}+N_{s}}\right\}$ with the transition rates satisfying:

$$
\left\{\begin{array}{l}
\operatorname{Pr}\{X(t+h)=j \mid X(t)=i\}=\lambda_{i j} h+o(h), \\
\operatorname{Pr}\{X(t+h)=i \mid X(t)=i\}=1-\lambda_{i i} h+o(h)
\end{array}\right.
$$

where $\lambda_{i i}=\sum_{i \neq j} \lambda_{i j}$.

To obtain the probability of the system being in a specific configuration, we are interested in finding the probabilities $P_{i j}(t)=\operatorname{Pr}\{X(t)=j \mid X(0)=i\}, t \geq 0$. These probabilities are obtained by solving the Kolmogorov forward equation given as:

$$
\dot{P}_{i j}=-\lambda_{i i} P_{i j}(t)+\sum_{y \neq j} P_{i y}(t) \lambda_{y j}
$$

Now, probability of being at configuration $j$ denoted by $\pi_{j}(t)$ is given by:

$$
\pi_{j}(t)=\operatorname{Pr}\{X(t)=j\}=\sum_{i} \operatorname{Pr}\{X(t)=j \mid X(0)=i\} \operatorname{Pr}\{X(0)=i\}=\sum_{i} P_{i j} \operatorname{Pr}\{X(0)=i\}
$$

Using the above equation and (29), it is concluded that:

$$
\dot{\pi}_{j}=-\lambda_{j j} \pi_{j}+\sum_{y \neq j} \pi_{y} \lambda_{y j}
$$

or in the matrix form:

$$
\dot{\pi}(t)=A \pi(t)
$$

where

$$
A_{i j}=\left\{\begin{array}{l}
-\lambda_{i i} \text { if } i=j \\
\lambda_{j i} \text { if } i \neq j
\end{array} .\right.
$$


Using the lattice of configurations, the set of possible configurations $\mathcal{F}$ is divided into three groups: Unstable configurations where their indices is collected in $U S$, Stable configurations with the index set $S T$, Admissible configurations with index set $A D$. Then, one can define different probabilistic measures based on the solutions of the equation $\dot{\pi}=A \pi$. Then the probability that the system is in a stable configuration at time $t$ is given by:

$$
P_{S T}(t)=\operatorname{Pr}\{X(t) \in S T\}=\sum_{j \in S T} \pi_{j}(t)
$$

and similarly the probability that the system performance is admissible is given by:

$$
P_{A D}(t)=\operatorname{Pr}\{X(t) \in A D\}=\sum_{j \in A D} \pi_{j}(t) .
$$

Therefore, the mean-time-to failure (MTTF) is calculated as:

$$
M T T F=\int_{0}^{\infty} P_{S T}(t) d t
$$

Similarly, the mean-time that with admissible performance is given as:

$$
M T A D=\int_{0}^{\infty} P_{A D}(t) d t .
$$

\subsection{Example}

We continue with example 1. It is assumed that the mission time that we are interested in is $10^{4}$ hours and the actuator failure rate is much smaller than the sensors. The sensor failure rates are identical and given as $\lambda_{a}=\lambda_{b}=\lambda_{c}=5 \times 10^{-5} h^{-1}$. By solving equations, the probability of being in the stable mode (being in a stable configuration) or in an unstable mode (being in an unstable configuration) over time is calculated which is depicted in Figure 5. Now, we assume that a redundant sensor for the first state is added which is denoted by $s_{1}^{\prime}$. This sensor is used when the sensor $s_{1}$ has failed. Therefore, the lattice of the system configurations would be as in Figure 4. Using this lattice and equation (32), the probability of being in a stable state in now computed which is shown in Figure 5. Comparing the results in rows of Figure 5 shows that the probability of being in a stable situation has now increased. To show the result clearly, we also consider a case where a redundant sensor for the third state is added. Using the same procedure $P_{S T}$ and $P_{U S}$ are computed. Figure 6 shows probability of being in the stable mode for these three configurations over time $\left[0,10^{4}\right]$. As can be seen, adding sensor $s_{1}^{\prime}$ gives the best result and increases the probability of being in the stable mode about $50 \%$ while adding $s_{3}^{\prime}$ increases this probability only by $14 \%$. 


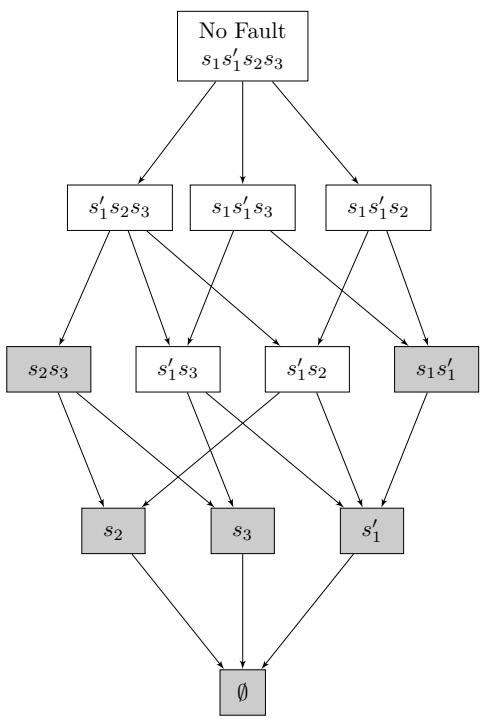

Figure 4: Lattice of system configurations for example 1 with redundant sensor for the first state 

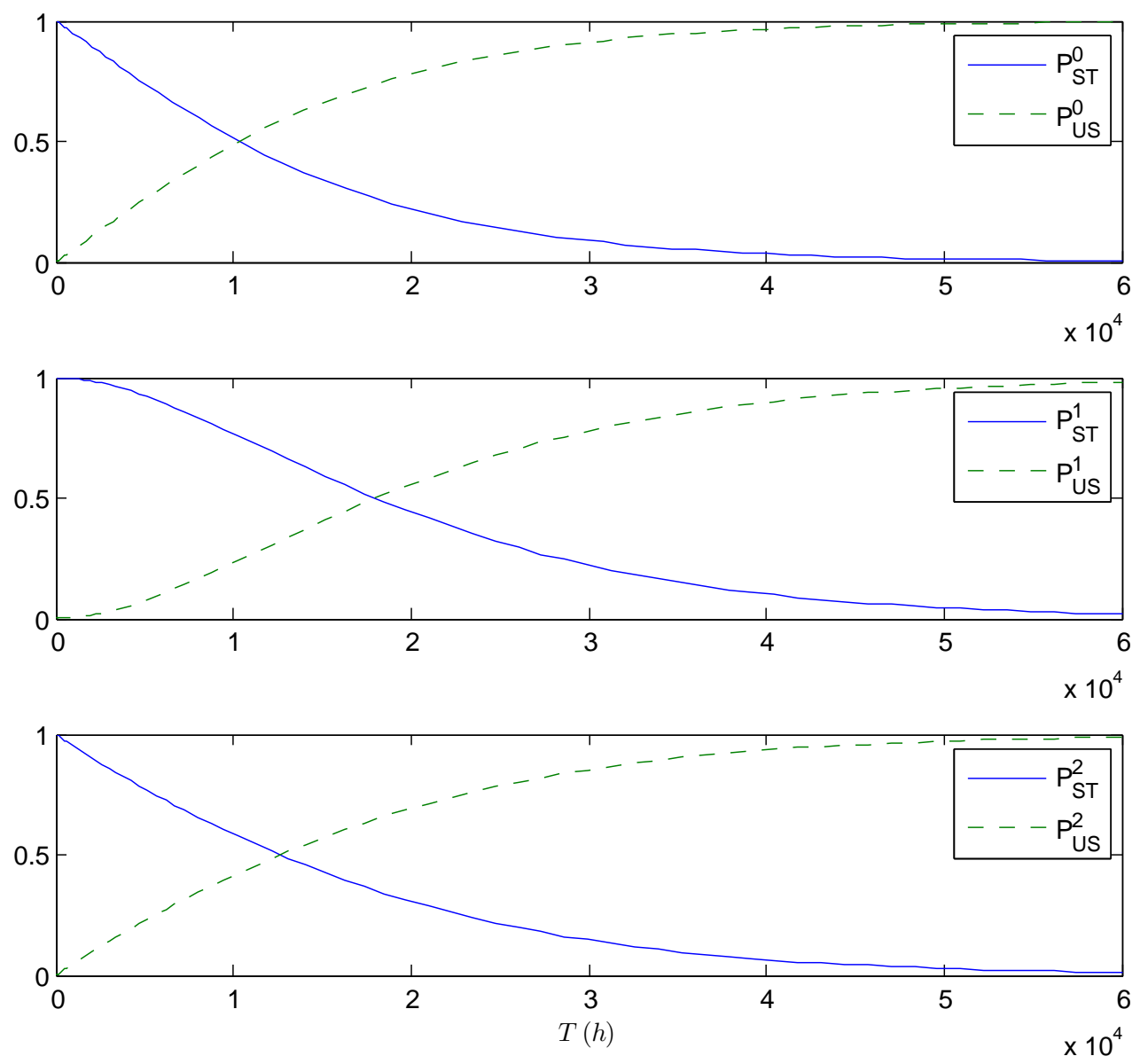

Figure 5: Probability of being in the stable $\left(P_{S T}\right)$ and unstable mode $P_{U S}$ with different configurations: (Top row) one sensor for each state $\left(s_{1} s_{2} s_{3}\right)$, (Middle row) a redundant sensor for the first measurement $\left(s_{1} s_{1}^{\prime} s_{2} s_{3}\right)$, (Bottom row) a redundant sensor for the third state $\left(s_{1} s_{2} s_{3} s_{3}^{\prime}\right)$ 


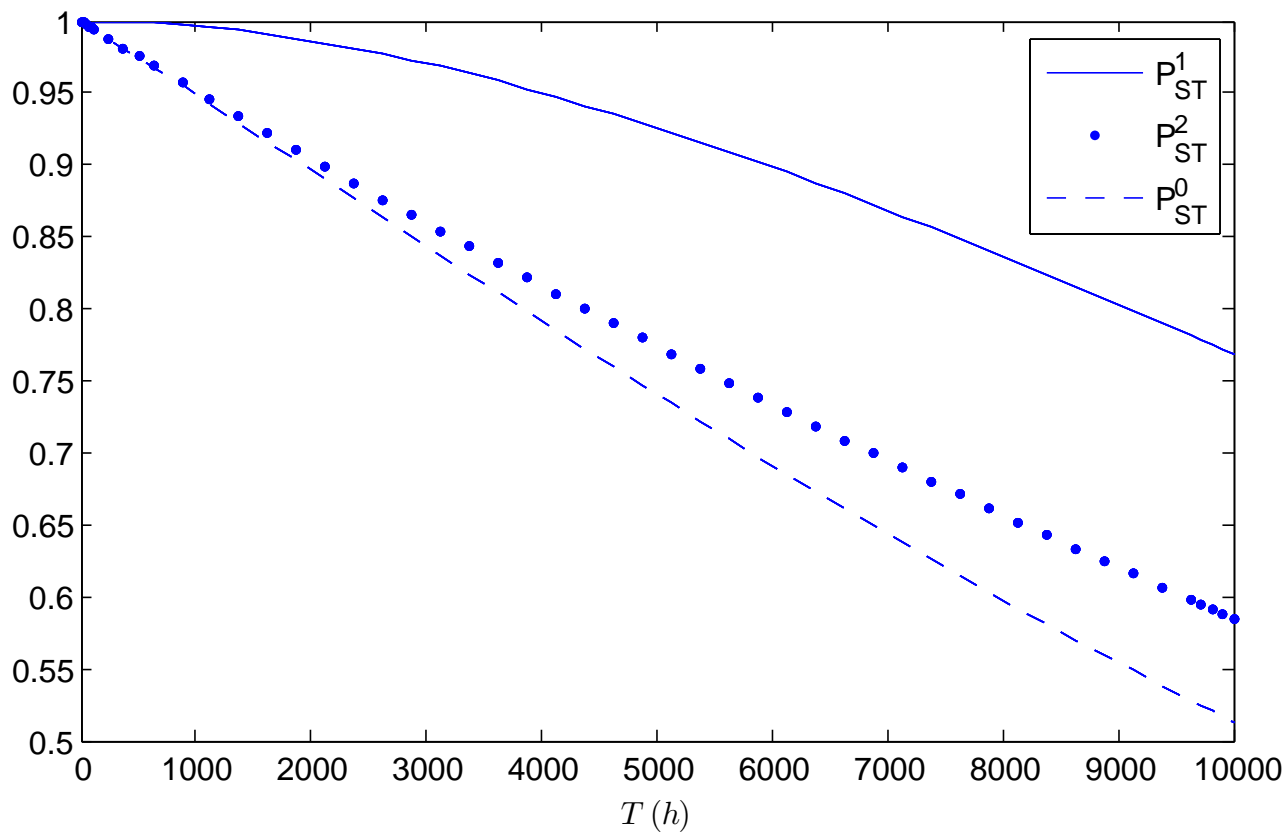

Figure 6: Probability of being in the stable mode with different configurations: (Top row) one sensor for each state $\left(s_{1} s_{2} s_{3}\right)$, (Middle row) a redundant sensor for the first measurement $\left(s_{1} s_{1}^{\prime} s_{2} s_{3}\right)$, (Bottom row) a redundant sensor for the third state $\left(s_{1} s_{2} s_{3} s_{3}^{\prime}\right)$.

\section{$5 \quad$ Reconfigurability analysis using $H_{\infty}$ performance}

In this section we define reconfigurability of a systems subject to fault based on $H_{\infty}$ performance. Consider the following discrete time piecewise affine system:

$$
\begin{aligned}
& x(k+1)=A_{i} x(k)+B_{i} u(k)+D_{i} w(k)+b_{i}, \\
& y(k)=C_{i} x(k), \\
& z(k)=C_{z i} x(k)+B_{z i} u(k)+D_{z i} w(k), \quad \text { for } x(k) \in \mathcal{R}_{i},
\end{aligned}
$$

where $x(k) \in \mathbb{R}^{n}$ is the state, $u(k) \in \mathbb{R}^{m}$ is the control input, $w(k) \in \mathbb{R}^{r}$ is the disturbance input, $z(k) \in \mathbb{R}^{q}$ is the controlled output, and $y(k) \in \mathbb{R}^{p}$ is the measured output. The output space partition and the faults are defined as before. Therefore, the faulty system is described by:

$$
\begin{aligned}
& x(k+1)=A_{i} x(k)+B_{i}^{f} u(k)+D_{i} w(k)+b_{i}, \\
& y(k)=C_{i}^{f} x(k), \\
& z(k)=C_{z i} x(k)+B_{z i} u(k)+D_{z i} w(k), \quad \text { for } x(k) \in \mathcal{R}_{i}
\end{aligned}
$$


A system subject to the fault $f$ is called reconfigurable if the faulty system is stabilizable by a static output feedback of the form:

$$
u(k)=K y(k),
$$

and the $H_{\infty}$ performance of the system is below a specified threshold $\gamma^{*}$. For a given real number $\gamma$, assuming $x(0)=0$, the exogenous signal is attenuated by $\gamma$, if for every integer $N \geq 0$ and for every $w \in L_{2}\left([0, N], R^{r}\right)$, the following inequality is satisfied:

$$
\sum_{k=0}^{N}\|z(k)\|^{2}<\gamma^{2} \sum_{k=0}^{N}\|w(k)\|^{2} .
$$

Theorem 4. The system (40) subject to fault $f$ with respect to admissibility threshold $\bar{\gamma}$ on the $H_{\infty}$ performance (45) is reconfigurable with a PWL static output feedback of the form (44) if there exist symmetric matrices $X_{i}=X_{i}^{T}>0$, matrices $U_{i}$, positive constants $\mu_{i l}, \gamma$, and $G_{i}$ with the following structure

$$
G_{i}=\left[\begin{array}{cc}
G_{11} & 0 \\
G_{i 21} & G_{i 22}
\end{array}\right]
$$

such that:

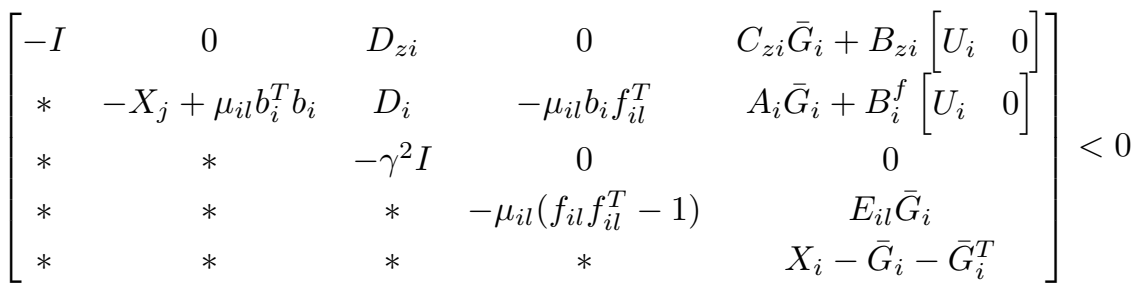

$$
\begin{aligned}
& \forall(i, j) \in \mathcal{S}, i \in \mathcal{I}_{1}, l=1, \cdots, l_{i},
\end{aligned}
$$

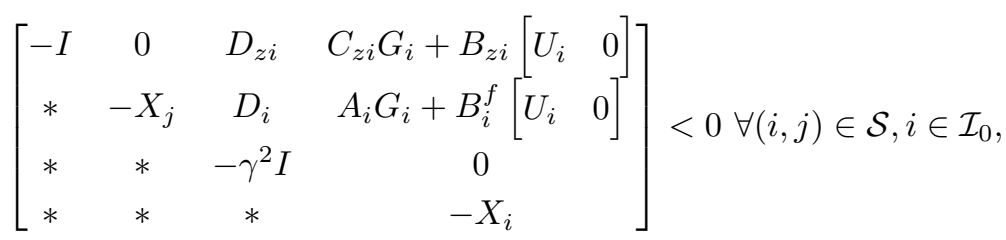

and

$$
\gamma<\bar{\gamma}
$$

, where $\bar{G}_{i}=T_{c i f} G_{i}$.

Proof. See A.3. 


\section{Examples}

\subsection{Example 2}

In this example, we consider the following PWL system:

$$
\begin{gathered}
A_{1}=\left[\begin{array}{lll}
0.4329 & 0.7604 & 0.2091 \\
0.2259 & 0.5298 & 0.3798 \\
0.5798 & 0.6405 & 0.7833
\end{array}\right] \quad A_{2}=\left[\begin{array}{lll}
0.6808 & 0.7942 & 0.0503 \\
0.4611 & 0.0592 & 0.4154 \\
0.5678 & 0.6029 & 0.3050
\end{array}\right] \\
B_{1}=B_{2}=I_{3 \times 3}, D_{1}=D_{2}=\mathbf{1}_{1 \times 3} \\
C_{1}=C_{2}=\left[\begin{array}{lll}
1 & 0 & 0 \\
0 & 1 & 0
\end{array}\right] C_{z 1}=C_{z 2}=I_{3 \times 3}, B_{z 1}=B_{1}, B_{z 2}=B_{2}, D_{z 1}=D_{z 2}=\mathbf{0}_{3 \times 1}
\end{gathered}
$$

We use $H_{\infty}$ performance of the system to analyze its reconfigurability as stated in theorem 4 . The result of the analysis on the lattice of configurations is shown in Figure 7. Each block represents a configuration where the corresponding component is failed. The graph starts with a situation where all components are healthy, at level 1 only one of the component is failed and so on. A grey block denotes that the configuration is not stabilizable (by a SOF). A white block denotes that the configuration is stabilizable. The corresponding $\gamma$ for the stabilizable configurations is also given. The results clearly indicates that ,assuming the failure rates are similar, sensor 2 is of crucial importance. If $s_{2}$ fails, then the system is not reconfigurable anymore. If the failure rates for the components are notably different, then the analysis should be augment with the probabilistic measures to make a better decision about the configuration selection.

\section{CONCLUSIONS}

We presented an approach for configuration selection for reconfigurable control of discrete time PWA systems. Reconfigurability is defined as both stability and admissibility of the upper bound on the performance of the system when it is stabilized using a static output feedback controller. We allow actuator and sensor failures and for the performance we consider both quadratic cost and $H_{\infty}$ performance associated with the system. Sufficient conditions for reconfigurability of a system subject to a fault with respect to a given threshold on the performance of the system are given in terms of LMIs. The upper bound is minimized by solving a convex optimization problem with LMI constraints. Through numerical examples we demonstrated how the proposed method can be used in the design phase to to decide about the optimal placement of sensors and actuators.

\section{References}

Boyd, S., Ghaoui, L.E., Feron, E., and Balakrishnan, V. (1994). Linear Matrix Inequalities in System and Control Theory. Society for Industrial Mathematics. 


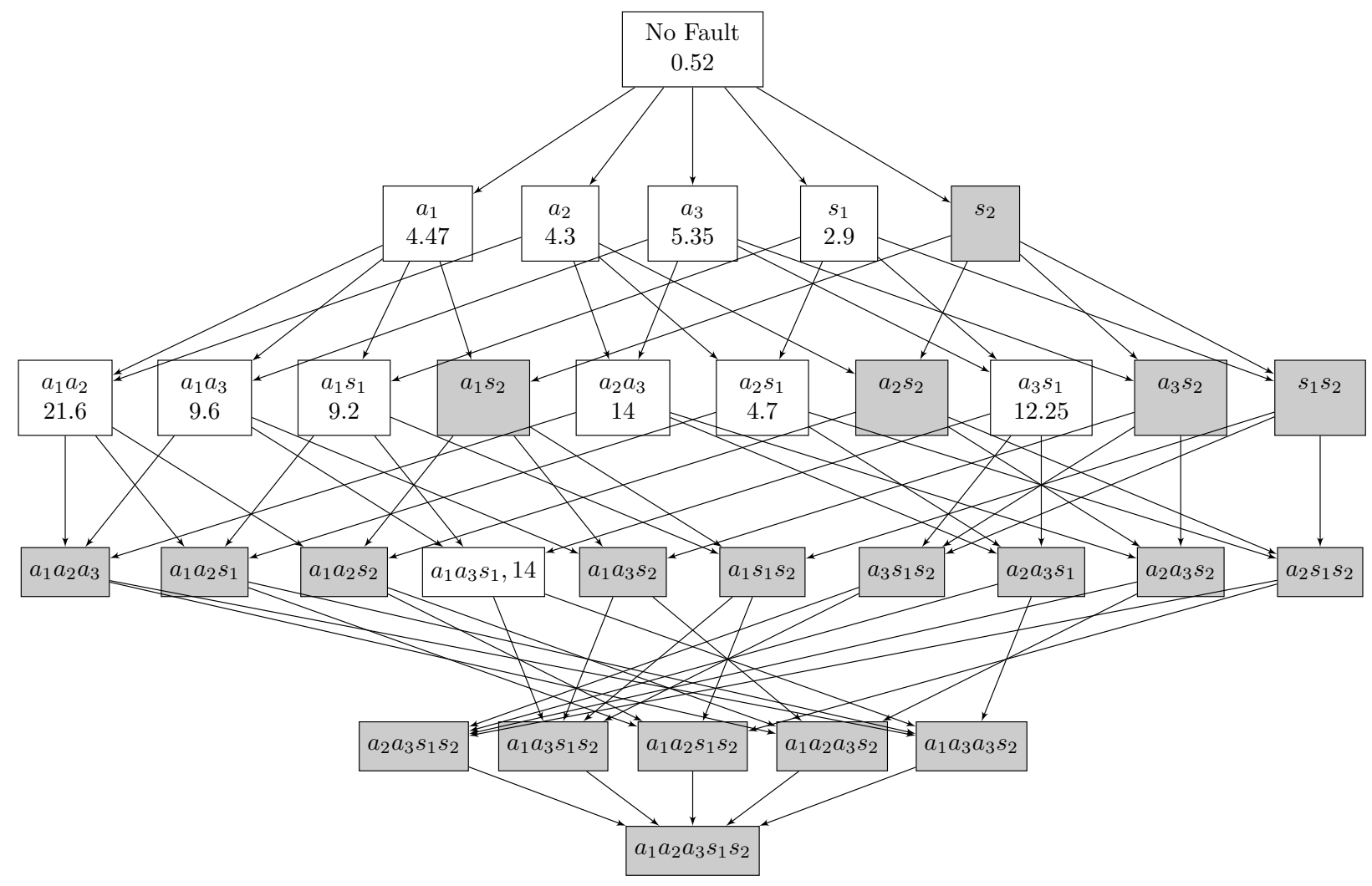

Figure 7: Lattice of system configurations for example 2, Each block represents a configuration where the corresponding component is failed.

Ferrari-Trecate, G., Muselli, M., Liberati, D., and Morari, M. (2003). A clustering technique for the identification of piecewise affine systems. Automatica, 39(2), $205-217$.

Frei, C., Kraus, F., and Blanke, M. (1999). Recoverability viewed as a system property. In European Control Conference. Karlsruhe, Germany.

Gonzalez-Contreras, B.M., Theilliol, D., and Sauter, D. (2009). Online Reconfigurability Evaluation for Actuator Fault Using Input/Output Data. In Proceedings of rth IFAC Symposium on Fault Detection, Supervision and Safety of Technical Processes, Safeprocess09.

Heemels, W., Schutter, B.D., and Bemporad, A. (2001). Equivalence of hybrid dynamical models. Automatica, 37(7), 1085-1091.

Johansson, M. (2003). Piecewise linear control systems. Springer-Verlag. 
Khelassi, A., Theilliol, D., and Weber, P. (2009). Reconfigurability analysis for reliable fault-tolerant control design. In Proceedings of 7th Workshop on Advanced Control and Diagnosis.

Ren, Z., Kroll, A., Sofsky, M., and Laubenstein, F. (2012). On identification of piecewise-affine models for systems with friction and its application to electro-mechanical throttles. In System Identification, volume 16, 1395-1400.

Richter, J.H., Heemels, W., van de Wouw, N., and Lunze, J. (2011). Reconfigurable control of piecewise affine systems with actuator and sensor faults: stability and tracking. Automatica.

Rodrigues, L. and Boyd, S. (2005). Piecewise-affine state feedback for piecewise-affine slab systems using convex optimization. Systems $\& 3$ Control Letters, 54(9), 835-853.

Staroswiecki, M. (2002). On reconfigurability with respect to actuator failures. In Proceedings of the 15th Triennial World Congress of IFAC.

Staroswiecki, M. (2003). Actuator faults and the linear quadratic control problem. In Proceedings of 42nd IEEE Conference on Decision and Control, volume 1, 959 - 965.

Staroswiecki, M., Commault, C., and Dion, J.M. (2012). Fault tolerance evaluation based on the lattice of system configurations. International Journal of Adaptive Control and Signal Processing, 26(1), 54-72.

Tabatabaeipour, S.M. and Bak, T. (2014). Robust observer-based fault estimation and accommodation of discrete-time piecewise linear systems. Journal of the Franklin Institute, 351(1), 277 - 295 . doi: http://dx.doi.org/10.1016/j.jfranklin.2013.08.021.

Tabatabaeipour, S.M., Gholami, M., Bak, T., and Schiøler, H. (2011). Reconfigurability of piecewise affine systems against actuator faults. In IFAC World Congress, volume 18, 4672-4677.

Tabatabaeipour, S.M., Gholami, M., Salahshoor, K., and Shaker, H. (2006). A clustering-based boundederror approach for identification of PWA hybrid systems. In 9th International Conference on Control, Automation, Robotics and Vision, 1-6.

Tabatabaeipour, S.M., Izadi-Zamanabadi, R., Bak, T., and Ravn, A.P. (2012). Passive fault-tolerant control of discrete-time piecewise affine systems against actuator faults. Internationl Journal of Systems Scinece, 43(11), 1985-1997.

van de Wouw, N. and Pavlov, A. (2008). Tracking and synchronisation for a class of PWA systems. Automatica, 44(11), 2909-2915.

Wu, N., Zhou, K., and Salomon, G. (2000). Control reconfigurability of linear time-invariant systems. Automatica, 36(11), 1767-1771.

Yang, Z. (2006). Reconfigurability analysis for a class of linear hybrid systems. In Proceedings of 6th IFAC safeprocess. 


\section{A Proofs}

\section{A.1 Proof of Theorem 1}

We consider a piecewise Lyapunov candidate function of the form $V\left(x(k)=x(k)^{T} P_{i} x(k), P_{i}>0\right.$ for $x(k) \in$ $\mathcal{X}_{i}$. To prove the stability we must have:

$$
V(x(k+1))-V(x(k))<0, \quad \forall(i, j) \in \mathcal{S} .
$$

We consider the general case where $x(k) \in \mathcal{R}_{i}$ and $x(k+1) \in \mathcal{R}_{j}$. First, we consider those switchings with $i \in \mathcal{I}_{1}$. To deal with the affine term, we will use the ellipsoidal approximation of regions. Substituting the state space equations of the closed loop system in (54) we get:

$$
\begin{gathered}
{\left[\left(A_{i}+B_{i}^{f} K C_{i}^{f}\right) x(k)+b_{i}\right]^{T} P_{j}\left[\left(A_{i}+B_{i}^{f} K C_{i}^{f}\right) x(k)+b_{i}\right]} \\
-x(k)^{T} P_{i} x(k)<0, \forall(i, j) \in \mathcal{S},
\end{gathered}
$$

which is equal to:

$$
\left[\begin{array}{c}
x(k) \\
1
\end{array}\right]^{T}\left[\begin{array}{cc}
\mathcal{A}_{i}^{T} P_{j} \mathcal{A}_{i}-P_{i} & * \\
b_{i}^{T} P_{j} \mathcal{A}_{i} & b_{i}^{T} P_{j} b_{i}
\end{array}\right]\left[\begin{array}{c}
x(k) \\
1
\end{array}\right]<0,
$$

where $\mathcal{A}_{i}=A_{i}+B_{i}^{f} K C_{i}^{f}$. The ellipsoidal approximation of $\mathcal{R}_{i}$ can be written as:

$$
\left[\begin{array}{c}
x(k) \\
1
\end{array}\right]^{T}\left[\begin{array}{cc}
E_{i l}^{T} E_{i l} & * \\
f_{i l}^{T} E_{i l} & f_{i l}^{T} f_{i l}-1
\end{array}\right]\left[\begin{array}{c}
x(k) \\
1
\end{array}\right] \leq 0, l=1, \ldots, \ell_{i},
$$

The condition $x(k) \in \mathcal{R}_{i}$ is relaxed to the above approximation. Using the S-procedure, see Boyd et al. (1994), the equation (56) is satisfied if there exist multipliers $\lambda_{i l}>0$ such that:

$$
(56)-\lambda_{i l}\left[\begin{array}{c}
x(k) \\
1
\end{array}\right]^{T}\left[\begin{array}{cc}
E_{i l}^{T} E_{i l} & * \\
f_{i l}^{T} E_{i l} & f_{i l}^{T} f_{i l}-1
\end{array}\right]\left[\begin{array}{c}
x(k) \\
1
\end{array}\right]<0 .
$$

This means that the following matrix inequality must be satisfied:

$$
\left[\begin{array}{cc}
\mathcal{A}_{i}^{T} P_{j} \mathcal{A}_{i}-P_{i} & \mathcal{A}_{i}^{T} P_{j} b_{i} \\
b_{i}^{T} P_{j} \mathcal{A}_{i} & b_{i}^{T} P_{j} b_{i}
\end{array}\right]-\lambda_{i l}\left[\begin{array}{cc}
E_{i l}^{T} E_{i l} & * \\
f_{i l}^{T} E_{i l} & f_{i l}^{T} f_{i l}-1
\end{array}\right]<0,
$$

which is equivalent to:

$$
\left[\begin{array}{cc}
-P_{i}-\lambda_{i l} E_{i l}^{T} E_{i l} & * \\
-\lambda_{i l} f_{i l}^{T} E_{i l} & -\lambda_{i l}\left(f_{i l}^{T} f_{i l}-1\right)
\end{array}\right]+\left[\begin{array}{c}
\mathcal{A}_{i}^{T} \\
b_{i}^{T}
\end{array}\right] P_{j}\left[\begin{array}{ll}
A_{i} & b_{i}
\end{array}\right]<0 .
$$

Applying Schur complement to the above equation we have:

$$
\left[\begin{array}{ccc}
-P_{i}-\lambda_{i l} E_{i l}^{T} E_{i l} & * & * \\
-\lambda_{i l} f_{i l}^{T} E_{i l} & -\lambda_{i l}\left(f_{i l}^{T} f_{i l}-1\right) & * \\
\mathcal{A}_{i} & b_{i} & -P_{j}^{-1}
\end{array}\right]<0 .
$$


Pre- and Post-multiplying the above equation with $\operatorname{diag}\left\{I,\left[\begin{array}{ll}0 & I \\ I & 0\end{array}\right]\right\}$, we get:

$$
\left[\begin{array}{ccc}
-P_{i}-\lambda_{i l} E_{i l}^{T} E_{i l} & * & * \\
\mathcal{A}_{i} & -P_{j}^{-1} & * \\
-\lambda_{i l} f_{i l}^{T} E_{i l} & b_{i}^{T} & -\lambda_{i l}\left(f_{i l}^{T} f_{i l}-1\right)
\end{array}\right]<0 .
$$

Using Schur complement, this is equal to:

$$
\begin{gathered}
{\left[\begin{array}{cc}
-P_{i}-\lambda_{i l} E_{i l}^{T} E_{i l} & * \\
\mathcal{A}_{i} & -P_{j}^{-1}
\end{array}\right]+} \\
{\left[\begin{array}{c}
-\lambda_{i l} E_{i l}^{T} f_{i l} \\
b_{i}
\end{array}\right] \lambda_{i l}^{-1}\left(f_{i l}^{T} f_{i l}-1\right)^{-1}\left[\begin{array}{ll}
-\lambda_{i l} f_{i l}^{T} E_{i l} & b_{i}^{T}
\end{array}\right]<0,}
\end{gathered}
$$

which is equal to:

$$
\begin{gathered}
{\left[\begin{array}{cc}
-P_{i}-\lambda_{i l} E_{i l}^{T} E_{i l} & * \\
\mathcal{A}_{i} & -P_{j}^{-1}
\end{array}\right]+} \\
{\left[\begin{array}{cc}
\lambda_{i l} E_{i l}^{T} f_{i l}\left(f_{i l}^{T} f_{i l}-1\right)^{-1} f_{i l}^{T} E_{i l} & * \\
-b_{i}\left(f_{i l}^{T} f_{i l}-1\right)^{-1} f_{i l}^{T} E_{i l} & \lambda_{i l}^{-1} b_{i}\left(f_{i l}^{T} f_{i l}-1\right)^{-1} b_{i}^{T}
\end{array}\right]<0 .}
\end{gathered}
$$

Using the matrix inversion Lemma, we have:

$$
\left(1-f_{i l}^{T} f_{i l}\right)^{-1}=1+f_{i l}^{T}\left(1-f_{i l} f_{i l}^{T}\right)^{-1} f_{i l} .
$$

The inequality (65) can be written as:

$$
\begin{gathered}
{\left[\begin{array}{cc}
-P_{i}-\lambda_{i l} E_{i l}^{T} E_{i l} & * \\
\mathcal{A}_{i} & -P_{j}^{-1}
\end{array}\right]+\left[\begin{array}{cc}
\lambda_{i l} E_{i l}^{T} E_{i l} & * \\
0 & -\lambda_{i l}^{-1} b_{i} b_{i}^{T}
\end{array}\right]+} \\
{\left[\begin{array}{c}
E_{i l}^{T} \\
-\lambda_{i l}^{-1} b_{i} f_{i l}^{T}
\end{array}\right] \lambda_{i l}\left(f_{i l} f_{i l}^{T}-I\right)^{-1}\left[\begin{array}{ll}
E_{i l} & -\lambda_{i l}^{-1} f_{i l} b_{i}^{T}
\end{array}\right]<0,}
\end{gathered}
$$

which, by using Schur complement, is equal to:

$$
\left[\begin{array}{ccc}
-P_{i} & * & * \\
\mathcal{A}_{i} & -P_{j}^{-1}-\mu_{i l} b_{i} b_{i}^{T} & * \\
E_{i l} & -\mu_{i l} f_{i l} b_{i}^{T} & -\mu_{i l}\left(f_{i l} f_{i l}^{T}-I\right)
\end{array}\right]<0
$$

where $\mu_{i l}=\lambda_{i l}^{-1}$. Replacing $\mathcal{A}_{i}$ by $A_{i}+B_{i}^{f} K C_{i}^{f}$, it is equivalent to:

$$
\left[\begin{array}{ccc}
-P_{i} & * & * \\
\left(A_{i}+B_{i}^{f} K C_{i}^{f}\right) & -P_{j}^{-1}-\mu_{i l} b_{i} b_{i}^{T} & * \\
E_{i l} & -\mu_{i l} f_{i l} b_{i}^{T} & -\mu_{i l}\left(f_{i l} f_{i l}^{T}-1\right)
\end{array}\right]<0,
$$


Post- and pre-multiply (69) by $\operatorname{diag}\left\{T_{c f i} G_{i}, I, I\right\}$ and its transpose, we get:

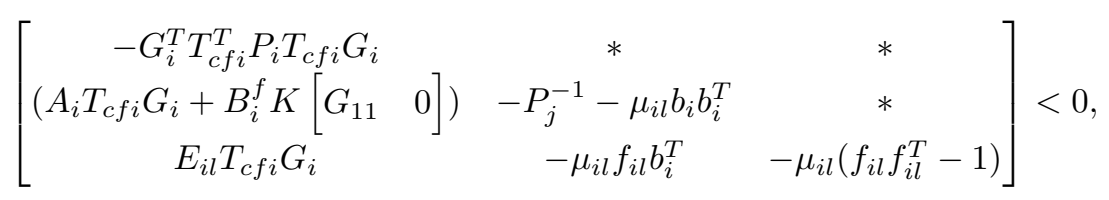

Using the fact that $G_{i}^{T} T_{c f i}^{T} P_{i} G_{i} T_{c f i} \geq T_{c f i} G_{i}+G_{i}^{T} T_{c f i}^{T}-P_{i}^{-1}$, and defining $U=K G_{11}$ we get the following condition as a sufficient condition for the satisfaction of the above inequality.

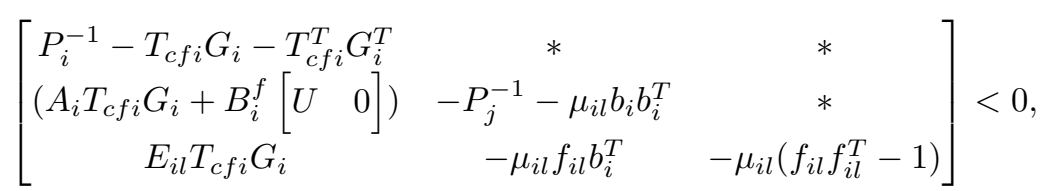

Define $X_{i}=P_{i}^{-1}$ and $\bar{G}_{i}=T_{c f i} G_{i}$ we get (13) as a sufficient condition for 54 .

For subsystems that contain the origin i.e. $i \in \mathcal{I}_{0}$, we have $f_{i l} f_{i l}^{T}-I<0$ which means that the LMI (13) is not feasible. For these subsystems the LMI (14) is considered and there is no need to include the region information. Therefore, the following matrix inequality must be satisfied:

$$
\left(A_{i}+B_{i}^{f} K C_{i}^{f}\right)^{T} P_{j}\left(A_{i}+B_{i}^{f} K C_{i}^{f}\right)-P_{i}<0 .
$$

Using Schur complement, the above inequality is equivalent to:

$$
\left[\begin{array}{cc}
-P_{i} & \left(A_{i}+B_{i}^{f} K C_{i}^{f}\right)^{T} \\
\left(A_{i}+B_{i}^{f} K C_{i}^{f}\right) & -P_{j}^{-1}
\end{array}\right]<0 .
$$

Post- and pre-multiply (73) by $\operatorname{diag}\left\{T_{c i} G_{i}, I, I\right\}$ and its transpose, we get:

$$
\left[\begin{array}{rc}
-G_{i}^{T} T_{c f i}^{T} P_{i} T_{c f i} G_{i} & * \\
\left(A_{i} T_{c f i} G_{i}+B_{i}^{f} K\left[\begin{array}{ll}
G_{11} & 0
\end{array}\right]\right. & -P_{j}^{-1}
\end{array}\right]<0,
$$

Using the fact that $G_{i}^{T} T_{c f i}^{T} P_{i} G_{i} T_{c f i} \geq T_{c f i} G_{i}+G_{i}^{T} T_{c f i}^{T}-P_{i}^{-1}$, and defining $U=K G_{11}$ we get the following condition as a sufficient condition for the satisfaction of the above inequality.

$$
\left[\begin{array}{cc}
P_{i}^{-1}-T_{c f i} G_{i}-T_{c f i}^{T} G_{i}^{T} & * \\
\left(A_{i} T_{c f i} G_{i}+B_{i}^{f}\left[\begin{array}{ll}
U & 0
\end{array}\right]\right) & -P_{j}^{-1}
\end{array}\right]<0,
$$

Define $X_{i}=P_{i}^{-1}$ and $\bar{G}_{i}=T_{c f i} G_{i}$ we get (13).

\section{A.2 Proof of Theorem 3}

We consider a piecewise Lyapunov candidate function of the form $V\left(x(k)=x(k)^{T} P_{i} x(k), P_{i}>0\right.$ for $x(k) \in$ $\mathcal{X}_{i}$. The condition to be satisfied is:

$$
\begin{aligned}
& V(x(k+1))-V(x(k))+x(k)^{T} Q_{i} x(k)+ \\
& x(k)^{T} C_{i}^{T} K_{i}^{T} R_{i} K_{i} C_{i} x(k)<0, \forall(i, j) \in \mathcal{S} .
\end{aligned}
$$


The proof of stability is very similar to the previous theorem except that to deal with the term $x(k)^{T} Q_{i} x(k)+$ $x(k)^{T} K_{i}^{T} R_{i} K_{i} x(k)$ we use the Schur complement two more times at the end of the proof. To prove that $(22)$ is satisfied we sum up (76) from $k=0$ to $k=\infty$, which results in:

$$
V(x(\infty))-V(x(0))+\Sigma_{0}^{\infty}\left(x^{T}(k) Q_{i} x(k)+u^{T}(k) R_{i} u(k)\right)<0
$$

Because $Q_{i}$ and $R_{i}$ are positive, hence $x(k)^{T} Q_{i} x(k)+x(k)^{T} C_{i}^{T} K_{i}^{T} R K_{i} C_{i} x(k) \geq 0$. Therefore, if (76) is satisfied the system is stable which means $V(x(\infty))=0$. As $V(x(0))=x(0)^{T} P_{i_{0}} x(0)$. Therefore we have:

$$
\sum_{k=0}^{\infty}\left(x^{T}(k) Q_{i} x(k)+u^{T}(k) R_{i} u(k)\right)<x^{T}(0) P_{i_{0}} x(0) .
$$

\section{A.3 Proof of Theorem 4}

The equations for the closed loop system are:

$$
\begin{aligned}
x(k+1) & =\mathcal{A}_{i} x(k)+D_{i} w(k)+b_{i}, \\
z(k) & =\mathcal{C}_{z i} x(k)+D_{z i} w(k),
\end{aligned}
$$

where $\mathcal{A}_{i}=A_{i}+B_{i} \Delta^{f} K_{i} \Lambda^{f} C_{i}$ and $\mathcal{C}_{z i}=C_{z i}+B_{z i} K_{i} \Lambda^{f} C_{i}$. We consider the following peicewise Lyapunov function:

$$
V(x(k))=x(k)^{T} P_{i} x(k), y(k) \in \mathcal{R}_{i}
$$

To prove that the induced $l_{2}$ norm of $w$ to the controlled output $z$ is less than $\gamma$, one must show that the following inequality holds:

$$
V(x(k+1))-V(x(k))+z^{T}(k) z(k)-\gamma^{2} w(k)^{T} w(k)<0 .
$$

Substituting (80) and the system equations (78) in the above equations we get:

$$
\left[\begin{array}{c}
x(k) \\
w(k) \\
1
\end{array}\right]^{T}\left\{\left[\begin{array}{c}
\mathcal{A}_{i}^{T} \\
D_{i}^{T} \\
b_{i}^{T}
\end{array}\right] P_{j}(*)+\left[\begin{array}{c}
\mathcal{C}_{i}^{T} \\
D_{z i}^{T} \\
0
\end{array}\right](*)+\left[\begin{array}{ccc}
-P_{i} & 0 & 0 \\
0 & -\gamma^{2} & 0 \\
0 & 0 & 0
\end{array}\right]\right\}\left[\begin{array}{c}
x(k) \\
w(k) \\
1
\end{array}\right]<0, \forall(i, j) \in \mathcal{S} .
$$

Using the regional information and the S-procedure we have:

$$
-\lambda_{i l}\left[\begin{array}{ccc}
0 & 0 & 0 \\
0 & E_{i l}^{T} E_{i l} & * \\
0 & f_{i l}^{T} E_{i l} & \left(f_{i l}^{T} f_{i l}-1\right)
\end{array}\right]+\left[\begin{array}{c}
D_{i}^{T} \\
\mathcal{A}_{i}^{T} \\
b_{i}^{T}
\end{array}\right] P_{j}(*)+\left[\begin{array}{c}
D_{z i}^{T} \\
\mathcal{C}_{i}^{T} \\
0
\end{array}\right](*)+\left[\begin{array}{ccc}
-\gamma^{2} & 0 & 0 \\
0 & -P_{i} & 0 \\
0 & 0 & 0
\end{array}\right]<0 \forall(i, j) \in \mathcal{S}
$$

Using Schur complement, the following inequality implies the above inequality:

$$
\left[\begin{array}{ccccc}
-I & 0 & D_{z i} & \mathcal{C}_{i} & 0 \\
* & -P_{j} & D_{i} & \mathcal{A}_{i} & b_{i} \\
* & * & \gamma^{2} I & 0 & 0 \\
* & * & * & -P_{i}-\lambda_{i l} E_{i l}^{T} E_{i l} & -\lambda_{i l} E_{i l}^{T} f_{i l} \\
* & * & * & * & -\lambda_{i l}\left(f_{i l}^{T} f_{i l}-1\right)
\end{array}\right]<0 .
$$


This is equal to

$$
\left.\left[\begin{array}{cccc}
-I & 0 & D_{z i} & \mathcal{C}_{i} \\
* & -P_{j}^{-1} & D_{i} & \mathcal{A}_{i} \\
* & * & \gamma^{2} I & 0 \\
* & * & * & -P_{i}-\lambda_{i l} E_{i l}^{T} E_{i l}
\end{array}\right]+\left[\begin{array}{c}
0 \\
b_{i} \\
0 \\
-\lambda_{i l} E_{i l}^{T} f_{i l}
\end{array}\right]\left[\lambda_{i l}\left(f_{i l}^{T} f_{i l}-1\right)\right]^{-1}\left[\begin{array}{c}
0 \\
b_{i} \\
0 \\
-\lambda_{i l} E_{i l}^{T} f_{i l}
\end{array}\right]\right]^{T}<\forall(i, j) \in \mathcal{S}
$$

Using the matrix inversion lemma as before, one can show that this is equal to:

$$
\left[\begin{array}{ccccc}
-I & 0 & D_{z i} & \mathcal{C}_{i} & 0 \\
* & -P_{j}^{-1}-\mu_{i l} b_{i}^{T} b_{i} & D_{i} & \mathcal{A}_{i} & -\mu_{i l} b_{i} f_{i l}^{T} \\
* & * & -\gamma^{2} I & 0 & 0 \\
* & * & * & -P_{i} & E_{i l}^{T} \\
* & * & * & * & -\mu_{i l}\left(f_{i l} f_{i l}^{T}-1\right)
\end{array}\right]<0 \forall(i, j) \in \mathcal{S}
$$

where $\mu_{i l}=\lambda_{i l}^{-1}$. We post- and pre-multiply the above equation with $\operatorname{diag}\left\{I, I, I,\left[\begin{array}{cc}0 & T_{c i} G_{i} \\ 1 & 0\end{array}\right]\right\}$ and its transpose respectively. Then we have:

$$
\left[\begin{array}{ccccc}
-I & 0 & D_{z i} & 0 & \mathcal{C}_{i} T_{c i} G_{i} \\
* & -P_{j}^{-1}-\mu_{i l} b_{i}^{T} b_{i} & D_{i} & -\mu_{i l} b_{i} f_{i l}^{T} & \mathcal{A}_{i} T_{c i} G_{i} \\
* & * & -\gamma^{2} I & 0 & 0 \\
* & * & * & -\mu_{i l}\left(f_{i l} f_{i l}^{T}-1\right) & E_{i l} T_{c i} G_{i} \\
* & * & * & * & -G_{i}^{T} T_{c i}^{T} P_{i} T_{c i} G_{i}
\end{array}\right]<0 \forall(i, j) \in \mathcal{S} .
$$

Notice that:

$$
\begin{aligned}
\mathcal{A}_{i} T_{c f i} G_{i}=\left(A_{i}+B_{i}^{f} K_{i} C_{i}^{f}\right) T_{c f i} G_{i}= & A_{i} T_{c f i} G_{i}+B_{i}^{f} K_{i} C_{i}^{f} T_{c f i} G_{i}=A_{i} T_{c f i} G_{i}+B_{i}^{f} K_{i}\left[\begin{array}{ll}
I & 0
\end{array}\right] G_{i}= \\
& A_{i} T_{c f i} G_{i}+B_{i}^{f}\left[\begin{array}{ll}
U & 0
\end{array}\right]
\end{aligned}
$$

where $U=K G_{11}$. Also, using a similar method we have:

$$
\mathcal{C}_{z i} T_{c f i} G_{i}=C_{z i} T_{c f i} G_{i}+B_{z i}\left[\begin{array}{ll}
U & 0
\end{array}\right]
$$

Using the fact that $G_{i}^{T} T_{c f i}^{T} P_{i} T_{c f i} G_{i} \geq G_{i}^{T} T_{c f i}^{T}+T_{c f i} G_{i}-P_{i}$ and defining $\bar{G}_{i}=T_{c f i} G_{i}$, then we have:

$$
\left[\begin{array}{ccccc}
-I & 0 & D_{z i} & 0 & C_{z i} \bar{G}_{i}+B_{z i}[U ⿻ 1 \\
* & -P_{j}^{-1}-\mu_{i l} b_{i}^{T} b_{i} & D_{i} & -\mu_{i l} b_{i} f_{i l}^{T} & A_{i} \bar{G}_{i}+B_{i}^{f}\left[\begin{array}{ll}
U & 0
\end{array}\right] \\
* & * & -\gamma^{2} I & 0 & 0 \\
* & * & * & -\mu_{i l}\left(f_{i l} f_{i l}^{T}-1\right) & E_{i l} \bar{G}_{i} \\
* & * & * & * & P_{i}^{-1}-\bar{G}_{i}-\bar{G}_{i}^{T}
\end{array}\right]<0 \forall(i, j) \in \mathcal{S},
$$


as a sufficient condition for (86). Define $X_{i}=P_{i}^{-1}$, we get (47). Proof of (48) is very similar except that there is no need to take into account the regional information. Once (47) and (48) are satisfied, there exist a PWL static output feedback that stabilizes the PWA faulty system and if $\gamma<\bar{\gamma}$, then the $H_{\infty}$ performance is admissible which means that the system subject to the fault $f$ is reconfigurable. 